\title{
Addressing spectroscopic quality of covariant density functional theory
}

\author{
A. V. Afanasjev $t$ \\ Department of Physics and Astronomy, Mississippi State University, Mississippi \\ State, Mississippi 39762, USA
}

\begin{abstract}
The spectroscopic quality of covariant density functional theory has been accessed by analyzing the accuracy and theoretical uncertainties in the description of spectroscopic observables. Such analysis is first presented for the energies of the single-particle states in spherical and deformed nuclei. It is also shown that the inclusion of particle-vibration coupling improves the description of the energies of predominantly single-particle states in medium and heavy-mass spherical nuclei. However, the remaining differences between theory and experiment clearly indicate missing physics and missing terms in covariant energy density functionals. The uncertainties in the predictions of the position of two-neutron drip line sensitively depend on the uncertainties in the prediction of the energies of the single-particle states. On the other hand, many spectroscopic observables in well deformed nuclei at ground state and finite spin only weakly depend on the choice of covariant energy density functional.

PACS numbers: 21.60.Jz, 21.10.Pc, 27.90.+b
\end{abstract}

\section{Introduction}

Among nuclear density functional theories (DFT), covariant density functional theory (CDFT) is one of most attractive since covariant energy density functionals (CEDF) exploit basic properties of QCD at low energies, such as symmetries and the separation of scales [1. They provide a consistent treatment of the spin degrees of freedom, they include the complicated interplay between the large Lorentz scalar and vector self-energies induced on the QCD level by the in-medium changes of the scalar and vector quark condensates [2, 3. Their local scalar and vector fields appear in the role of local relativistic Kohn-Sham potentials [4, 5. This class of successful scalar-vector mean field models can also be considered as a phenomenological realization of chiral effective field theories [6, 7]. The CEDF is approximated with powers and gradients of auxiliary meson fields or nucleon densities. The building of the covariant energy density functional in the context of effective field theory allows error estimates to be made, provides a power counting scheme which separates long- and short-distance dynamics, and therefore, removes model dependences from self-consistent mean field approach [8].

$\ddagger$ The correspondence should be addressed to afansjev@erc.msstate.edu 
In addition, relativistic treatment of the DFT problems offers substantial advantages over non-relativistic approach. First of all, the spin-orbit interaction emerges in a natural way in the CDFT [9, 10] and the results of the calculations are close to experiment [11, 12]. On the contrary, spin-orbit interaction is always parametrized in non-relativistic DFT [13. Second, covariant energy density functionals include nuclear magnetism [14, i.e. a consistent description of currents and time-odd mean fields important for odd-mass nuclei [15], the excitations with unsaturated spins, magnetic moments [16] and nuclear rotations [17, 18]. Because of Lorentz invariance no new adjustable parameters are required for the time-odd parts of the mean fields in CDFT. The effects of nuclear magnetism are most pronounced in rotating nuclei [17, 18]. The fact that the properties of rotating nuclei are well described in the CDFT calculations (see Refs. [19, 20] and references therein and Sect. 4 in this paper) strongly suggests that the effects of nuclear magnetism are correctly (as compared with experiment) reproduced. Moreover, they show only weak dependence on CEDF [15, 18]. These are important features which decrease model dependence of some spectroscopic observables (such as the moments of inertia). In contrast, several prescriptions (native, gauge and Landau [21]) exist for the description of time-odd mean fields in non-relativistic Skyrme DFT. As a consequence, their impact on physical observables such as binding energies of one-quasiparticle configurations of odd-mass nuclei [21] and the moments of inertia [22] is not uniquely defined.

Of course, at present, all attempts to derive CEDF's directly from the bare forces [23, 24, 25, 26] do not reach the required accuracy; the same is true also for nonrelativistic EDF's [27]. Even for most microscopically based CEDF DD-ME $\delta$ [28], four parameters are fitted to finite nuclei. Considering phenomenological content of modern EDF's, it is important to estimate theoretical uncertainties in the EDF parameters and in the description of physical observables. It was suggested in Refs. [29, 30] to use the methods of information theory for that purpose. These uncertainties come from the selection of the form of EDF as well as from the fitting protocol details, such as the selection of the nuclei under investigation, the physical observables, or the corresponding weights. Some of them are called statistical errors and can be calculated from a statistical analysis during the fit, others are systematic errors, such as for instance the form of the EDF under investigation, which are much more difficult to estimate because of possible missing physics [30]. This is especially true because of the current bias on the use of bulk properties (masses, radii, neutron skins) in the fit of the non-relativistic and covariant EDF's [31]. For example, spectroscopic (single-particle) information is never used in the fit of CEDF's. On the contrary, the limited information on the splitting of spin-orbit doublets is always employed in the fit of non-relativistic EDF's.

The current paper aims on a review of spectroscopic quality of CDFT. In that respect it is useful to recall the definition of "nuclear spectroscopy". There are several definitions available, and the one quoted below is a good representative example. According to Ref. [32], "Nuclear Spectroscopy is a branch of nuclear physics that 
is concerned with the study of the discrete spectrum of nuclear states, namely, with the determination of energy, spin, parity, isotopic spin, and other quantum characteristics of the nucleus in the ground and the excited states." Experimentally, these quantities are obtained by measuring either the $\gamma$-transitions between the states in the same nucleus or different decays $\left(\alpha, \beta^{+}, \beta^{-}\right.$etc) between the states in different nuclei. Thus, in the opinion of the author, this definition has to be extended by adding "and the transitions and decays between the states" at its end.

This definition of "nuclear spectroscopy" is presented here because much narrower definition, focused only on the accuracy of the description of the single-particle states in odd-mass nuclei, is frequently used in theoretical community when spectroscopic quality of specific theory is discussed. This narrow definition ignores collective excitations such as rotations and surface vibrations leading to rotational and vibrational bands; they are also studied by nuclear spectroscopy. In the present paper, the author will use the broad definition of "nuclear spectroscopy" when discussing spectroscopic quality of CDFT.

The analysis of theoretical uncertainties relies on statistical methods. The application of such methods is more complicated for spectroscopic physical observables than for ground state observables due to a number of reasons. First, such observables (for example, the moment of inertia which describes the evolution of rotational band and the $\gamma$-transitions between the $I$ and $I+2$ members of the band with spin) depend on external parameter (rotational frequency) so the calculations have to performed for a set of the values of this parameter. In addition, they have to be calculated in threedimensional computer codes which are numerically time-consuming. Second, time-odd mean fields have to be taken into account for the calculations of the energies of one(quasi)particle states in odd-mass spherical [11] and deformed [15, 33] nuclei and the moments of inertia [34, 17, 18] of nuclear configurations. Third, the blocking procedure has to be employed in odd-mass nuclei. However, it is frequently numerically unstable (see Ref. [33] and the discussion in Sect. V of Ref. [20]).

It is very difficult to perform the analysis of statistical errors on a global scale since the properties of transitional and deformed nuclei have to be calculated repeatedly for different variations of original EDF. Thus, such statistical analysis has been performed mostly for spherical nuclei [29, 35] or selected isotopic chains of deformed nuclei [36]. In Ref. [31, a global analysis of theoretical uncertainties for the ground state observables of even-even nuclei has been performed in the RHB calculations with four state-of-the-art CEDF's. However, these uncertainties are only crude approximation to the systematic theoretical errors discussed in Ref. [30] because of (i) limited choice of CEDF's and (b) possible similar missing physics in these functionals. It is clear that similar global analysis is not possible for spectroscopic observables because of the complexity of their calculations. However, as will be shown below the estimate of theoretical uncertainties and important physical conclusions related to spectroscopic physical observables can be obtained employing smaller set of data.

The paper is organized as follows. The accuracy of the description of predominantly single-particle states in spherical nuclei will be discussed in Sec. 2, In Sect. 3, I will 
analyse theoretical uncertainties in the description of deformed single-(quasi)particle states. The impact of the imperfections in the single-particle structure on the spectroscopic observables in rotating nuclei will be considered in Sect. 4. Sect. 5 is dedicated to the analysis of how the uncertainties in the description of the single-particle structure affect the predictions for the position of two-neutron drip line. Finally, Sect. 6] summarizes the results of this work.

\section{Single-particle states in spherical nuclei}

The experimental data on predominantly single-particle states in odd-mass spherical nuclei neighbouring to doubly magic nuclei has frequently been compared with model calculations on the mean field level. Let me give few examples; the list is definitely not complete. Such comparisons are performed in ${ }^{208} \mathrm{~Pb}$ for the NL1, NL3 and NLSH CEDF's in Fig. 20 of Ref. [37]), for the NL3, NL-Z, NL-Z2, NL-VT1 CEDF's in Fig. 1 of Ref. [12, for the DD-PC1 CEDF in Fig. 18 in Ref. [38], and for the PC-PK1, DD-PC1, PC-F1, PC-LA and NL3* CEDF's in Fig. 6 of Ref. [39]). For ${ }^{132} \mathrm{Sn}$ such comparisons are presented for DD-PC1 in Fig. 18 of Ref. [38] and for the PC-PK1, DD-PC1, PC-F1, PC-LA and NL3* CEDF's in Fig. 6 of Ref. [39]. These results lead to the following observations:

- the difference between the energies of some spherical subshells obtained with different CEDF's can come close to $2 \mathrm{MeV}$,

- the energies of some subshells may not be so much affected by the selection of CEDF,

- as a consequence of these two observations, the relative energies of different spherical subshells strongly depend on the CEDF,

- the existence of large shell gaps does not depend on CEDF, however, their size is CEDF dependent,

- the existence of smaller shell gaps and their size are strongly CEDF dependent.

As illustrated in Fig. 8 below, such features are also valid for nuclei at the neutron-drip line.

In general, it is not so difficult to perform the analysis of the statistical errors in the model predictions of the energies of the single-particle states of spherical nuclei at the mean field level in the spirit of Ref. [30] (see, for example, Fig. 9] below). However, it is already clear that such an analysis will provide only limited guidance because of two reasons. First, the states in odd-mass nuclei are strongly affected by coupling with vibrations [11, 40]. Second, there are systematic differences between different classes of the models which, for example, lead to systematic differences in model predictions for superheavy nuclei (see discussion below).

Fig. 1 shows that on average the inclusion of particle-vibration coupling (PVC) substantially improves the description of the spectra in ${ }^{132} \mathrm{Sn}$. A systematic analysis 


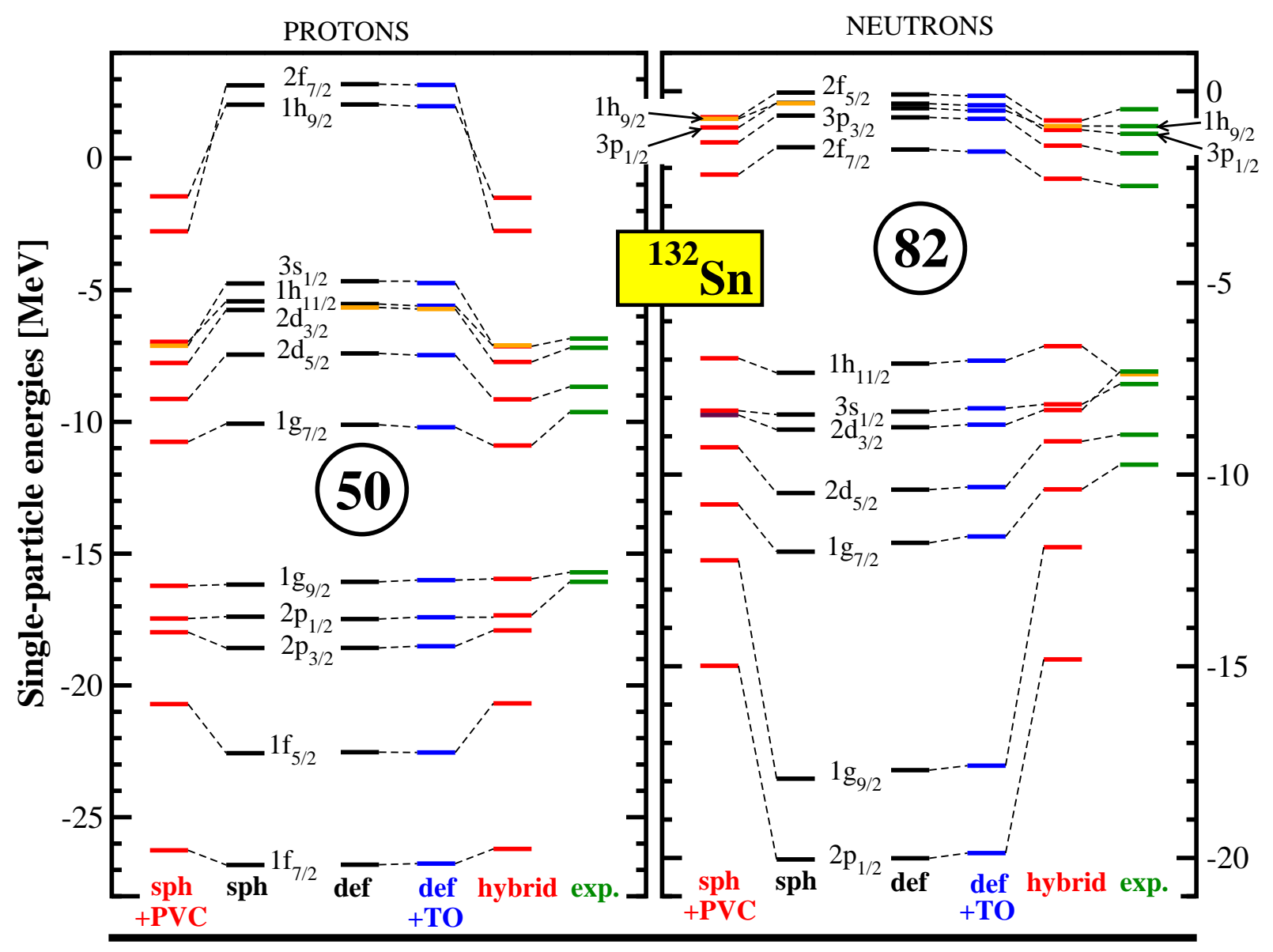

Figure 1. Spectra of ${ }^{132} \mathrm{Sn}$ and its neighboring odd nuclei. Column 'sph' shows the single-particle spectra obtained in spherical CDFT calculations of ${ }^{132} \mathrm{Sn}$. Column 'sph+PVC' shows the spectra obtained in spherical calculations within the PVC model. Columns "def", "def+TO", "hybrid" and "exp" show one-nucleon separation energies [11. Column "def" is based on the results of triaxial CRMF calculations with no time-odd mean fields. These fields are included in the calculations the results of which are shown in column "def+TO". The corrections due to PVC are added in column "hybrid". In order to distinguish overlapping levels, orange and then maroon colors are used for the levels in addition to their standard color used in a given column. From Ref. [11.

of Ref. [11] performed within the relativistic PVC model with the NL3* CEDF [41] covers ${ }^{56} \mathrm{Ni},{ }^{132} \mathrm{Sn}$ and ${ }^{208} \mathrm{~Pb}$. For these nuclei, average deviations per state $\Delta \varepsilon$ between calculated and experimental energies of the predominantly single-particle states are shown in Table 1. They are defined as

$$
\Delta \varepsilon=\frac{\sum_{i=1}^{N}\left|\varepsilon_{i}^{t h}-\varepsilon_{i}^{e x p}\right|}{N}
$$

where $N$ is the number of the states with known experimental single-particle energies, and $\varepsilon_{i}^{t h}\left(\varepsilon_{i}^{e x p}\right)$ are calculated (experimental) energies of the predominantly single-particle states. One can see that the inclusion of PVC substantially improves the description of the single-particle states in ${ }^{132} \mathrm{Sn}$ and ${ }^{208} \mathrm{~Pb}$. The same result has also been obtained in 
the ${ }^{208} \mathrm{~Pb}$ calculations with the NL3 CEDF [40]. On the contrary, PVC introduces no (small) improvement in the description of the proton (neutron) single-particle states of ${ }^{56} \mathrm{Ni}$.

Table 1. Average deviations per state $\Delta \varepsilon$ between calculated and experimental energies of the single-particle states for a proton (neutron) subsystem of a given nucleus. The results obtained in the "def+TO" and "hybrid" calculational schemes are shown (see caption of Fig. 1 and Ref. [1] for details).

\begin{tabular}{|c|c|c|}
\hline Nucleus/subsystem & $\Delta \varepsilon_{\text {def }+ \text { TO }}[\mathrm{MeV}]$ & $\Delta \varepsilon_{\text {hybrid }}[\mathrm{MeV}]$ \\
\hline${ }^{56} \mathrm{Ni} /$ proton & 0.76 & 0.77 \\
${ }^{56} \mathrm{Ni} /$ neutron & 0.89 & 0.71 \\
${ }^{132} \mathrm{Sn} /$ proton & 1.02 & 0.68 \\
${ }^{132} \mathrm{Sn} /$ neutron & 0.89 & 0.39 \\
${ }^{208} \mathrm{~Pb} /$ proton & 1.53 & 0.84 \\
${ }^{208} \mathrm{~Pb} /$ neutron & 1.00 & 0.47 \\
\hline
\end{tabular}

One can ask a question what is a reason for a such different behavior of PVC in light and medium/heavy nuclei. Note that similar behavior is also observed in Skyrme PVC calculations in which the PVC does (not) improve the description of predominantly single-particle states in medium/heavy (light) nuclei [42]. One of possibilities is related to the fact that contrary to medium $\left({ }^{132} \mathrm{Sn}\right) /$ heavy $\left({ }^{208} \mathrm{~Pb}\right)$ nuclei the lighter nuclei are characterized by soft potential energy surfaces. For such nuclei, the description of collective phonons within random phase approximation may be not adequate and more sophisticated methods such as generator coordinate method may be required [43].

This discussion clearly shows that single-particle observables are more complicated than bulk ones (such as masses, radii, neutron skin thicknesses etc) for which statistical error analysis is relatively simple [29, 30]. A necessary condition for an analysis of systematic errors is statistical independence of EDF's under consideration [30]. However, this condition is not satisfied in modern DFT's. The dominance of bulk observables and the ignorance of single-particle observables in the fitting protocols of EDF's leads to the bias towards former observables and possible missing physics and terms of EDF's. Different model assumptions also contribute to that.

The region of superheavy nuclei is a clear example where model biases (missing terms of EDF's and missing physics) should be analysed in detail and addressed before the analysis of statistical errors is undertaken. This is illustrated by the fact that the centers of the island of stability are located at different shell gaps in different models. For example, it is located at $Z=120$ and $N=172$ in most of CEDF's [12. Although the $N=172$ gap is preferred, neutron gap at $N=184$ cannot be excluded [44, 45]. Similar conclusion has also been reached in the relativistic PVC calculations of Refs. [11, 46]. On the other hand, non-relativistic Skyrme DFT favors $Z=126$ and $N=184$ and macroscopic+microscopic method favors $Z=114$ and $N=184$ [12]. 


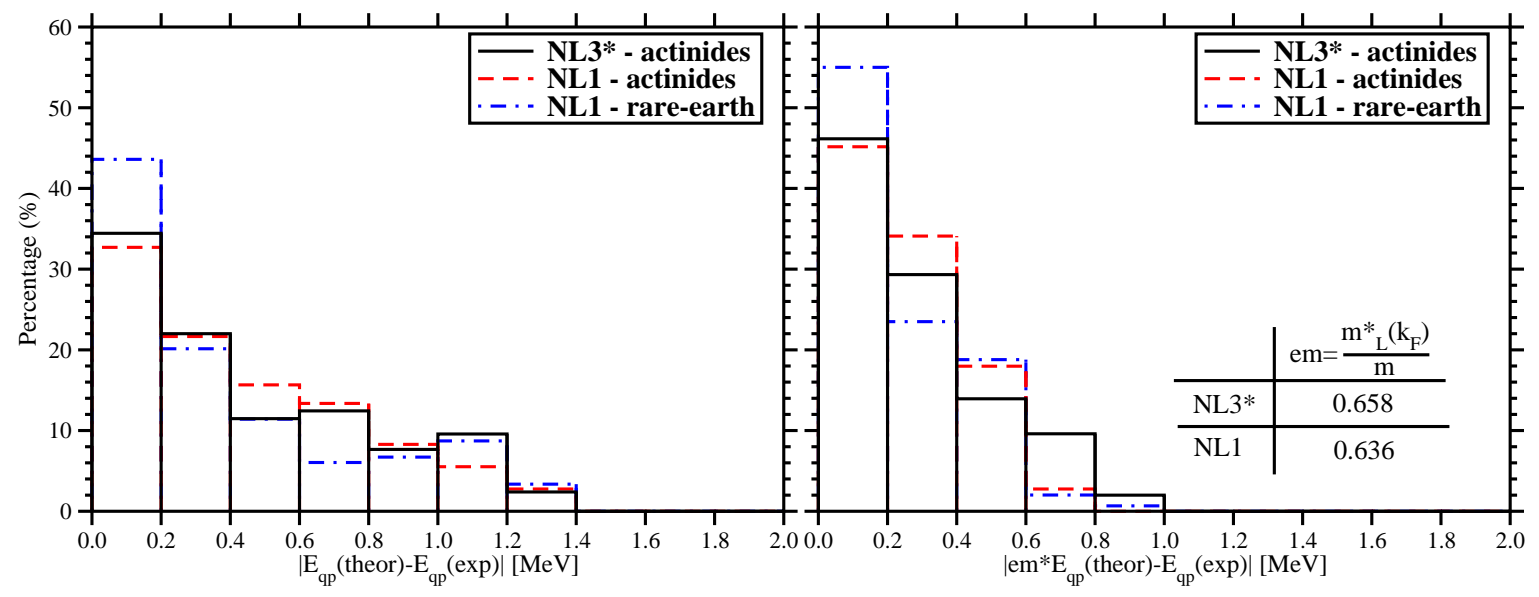

Figure 2. (left panel) The distribution of the deviations of the calculated energies $E_{q p}($ theor $)$ of one-quasiparticle states from experimental ones $E_{q p}(\exp )$. The vertical axis shows the percentage of the states which deviate from experiment by the energy deviation range (the width of bar) specified on horizontal axis. (right panel) The same as in left panel, but for the case when the energy scale of theoretical spectra is corrected for low Lorentz effective mass. Based on Figs. 2 and 3 of Ref. [33.

\section{Single-particle states in deformed nuclei}

An essential difference between the phenomenological models based on the WoodsSaxon or Nilsson potentials and self-consistent DFT calculations is the fact that the phenomenological potentials are fitted to experimental single-particle energies. As a consequence, they well describe the single-particle spectra in deformed systems. On the contrary, no single-particle information is used in the fit of CEDF's. In the nonrelativistic EDF's, the strength of the spin-orbit force is typically fitted to experimental data on spin-orbit splittings.

Despite extensive use of the DFT's to the description of nuclear phenomena, only recently few attempts to understand the accuracy of the description of the singleparticle spectra in deformed systems within the DFT framework have been undertaken. Restricted in scope investigations of experimental spectra in deformed odd nuclei have been performed in Skyrme [21] and Gogny [47] DFT. A statistical analysis of Ref. [33], performed in the CDFT with NL1 and NL3* CEDF's, represents the most extensive attempt to understand the systematic errors in the description of deformed one-quasiparticle states.

A statistical analysis [33] of the discrepancies between calculated and experimental energies of one-quasiparticle states in the ground state minimum is presented in the left panel of Fig. 3. One can see that in the actinide region only approximately $33 \%$ of one-quasiparticle states are described with an accuracy better than $200 \mathrm{keV}$, and approximately $22 \%$ with an accuracy between 200 and $400 \mathrm{keV}$ in the NL3* and NL1 CEDF's. The percentage of the states for a given range of deviations gradually decreases with increasing deviation between experiment and calculations. However, for some states the deviation of the calculated energy from experiment exceeds $1 \mathrm{MeV}$ and can 


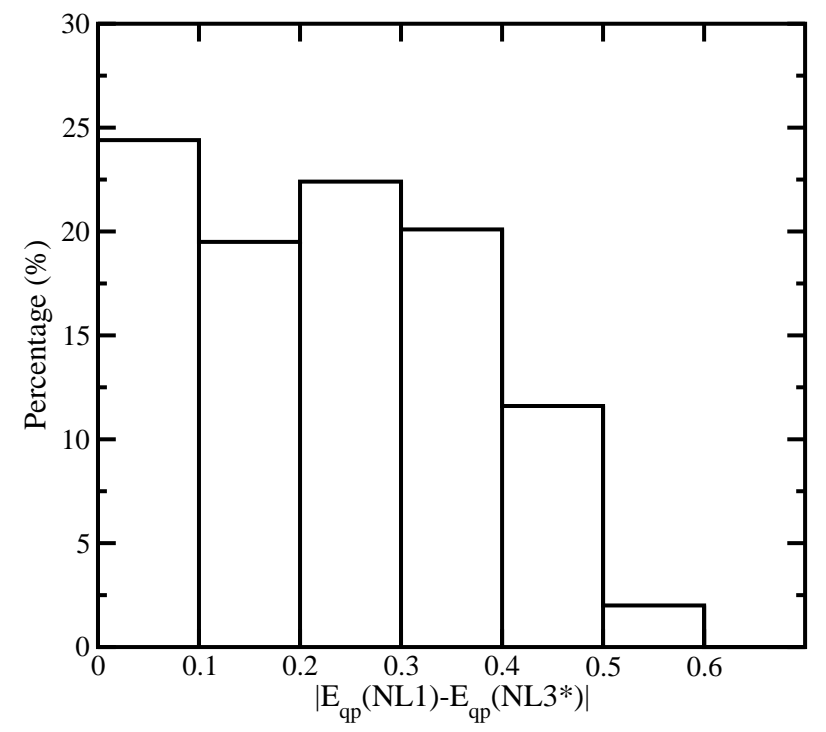

Figure 3. The distribution of the differences between the energies $E_{q p}$ of onequasiparticle states obtained in the RHB calculations with the NL1 and NL3* CEDF's. The vertical axis shows the percentage of the states which fall into the energy difference range (the width of the bar) specified on horizontal axis.

be close to 1.4 MeV. Fig. 3 also shows that with the NL1 CEDF the 1-qp energies in odd-proton rare-earth nuclei are somewhat better described as compared with actinide region. Otherwise, the distribution histograms for the deviations are similar in both regions and for both parametrizations.

The distribution of the differences between the energies $E_{q p}$ of one-quasiparticle states obtained in the RHB calculations with the NL1 and NL3* CEDF's is presented in Fig. 3. One can see that substantial differences in the description of one-quasiparticle states exist between employed CEDF's. The fact that these differences are smaller than those presented in Fig. 9 below for spherical nuclei are due to two facts. First, only two CEDF's are used in Fig. 3, while substantially larger set of ten CEDF's is used in Fig. 9. Second, while one-quasiparticle energies defined with respect of the energy of the ground state in odd-mass nucleus are used in Fig. 3 , the absolute single-particle energies are used in the creation of Fig. 9.

It is clear that the spectroscopic quality of the description of the single-particle spectra of the current generation of the DFT models (both relativistic and nonrelativistic ones) is lower than the one achievable in the macroscopic+microscopic (MM) method. In part, this is a consequence of different philosophies realized in the DFT and MM methods. It is well known that experimental "single-particle" states are not meanfield states; their wave functions are fragmented and always contain the admixtures from vibrational phonons. In odd mass nuclei, the weights of these admixtures are generally low for ground states but increase with increasing excitation energy of the level relative to the ground state [48, 49]. By fitting the parameters of phenomenological potentials to the energies of dominant single-particle states, the MM models effectively include vibrational corrections into these potentials but only on the level of the energies and not 
on the level of the wavefunctions. As a consequence, these potentials are characterized by an effective mass of the nucleon at the Fermi level $m^{*}\left(k_{F}\right) / m \approx 1.0$ which reproduces experimental level density. However, for these potentials the inclusion of the coupling to vibrations will lead to double counting of vibrational contribution in the energies and an effective mass of $m^{*}\left(k_{F}\right) / m \approx 1.4[50$.

On the contrary, single-particle levels are not adjusted to experiment in DFT's since their functionals are fitted mainly to bulk and neutron matter properties. As a consequence, most of them, in particular Gogny and relativistic functionals, are characterized by low effective mass of the nucleon (the Lorentz mass for the case of CDFT [51]), and calculated single-particle states do not effectively include vibrational corrections. A low effective mass leads to a stretching of the theoretical single-particle energy scale as compared with experiment, and, thus, to larger deviations between theory and experiment for deformed one-quasiparticle states (left panel of Fig. 3). To cure this problem one should go beyond the mean field approximation and supplement CDFT by particle-vibrational coupling (PVC). So far, this has been done only in spherical nuclei (see discussion in Sect. 2), for which it was shown that in the presence of PVC (i) calculated spectra of dominant single-particle states compress and come closer to experimental ones and (ii) effective mass of the nucleon comes closer to 1.

A similar compression of calculated spectra is expected also in deformed nuclei. However, so far, no PVC model based on the DFT framework has been developed for such nuclei. The analysis of Ref. [33] suggests that on average the expected compression of single-particle spectra can be achieved via a rescaling of one-quasiparticle (1-qp) energies by the Lorentz effective mass. The impact of such an energy rescaling on the distribution of the deviations between theory and experiment is shown in the right panel of Fig. 3, more than $75 \%$ of the states are described with an accuracy better than $400 \mathrm{keV}$. This is a typical accuracy of the description of the energies of deformed 1-qp states within phenomenological potentials [52, 53]. Although this energy rescaling is somewhat schematic and assumes that the effect of PVC is identical in spherical and deformed nuclei, it clearly illustrates that PVC, leading to an increase of the effective mass, could also improve the description of experimental spectra as compared with mean field results.

\section{Rotational structures}

Considering existing inaccuracies in the description of the energies of the single-particle states, it is important to understand how they affect other physical observables of interest, especially the ones of collective nature in which many single-particle states contribute. It turns out that these inaccuracies do not affect appreciably many collective observables in the situations when potential energy surface as a function of collective variable is well developed. For example, the fission barriers in actinides are described accurately despite existing uncertainties in the description of the single-particle states and low effective mass of nucleon [54, 55, 56, 57]. Moreover, the CDFT theory is the only 
DFT theory which provides such level of accuracy without a fit of the EDF parameters to the fission barriers or fission isomer energies [58].

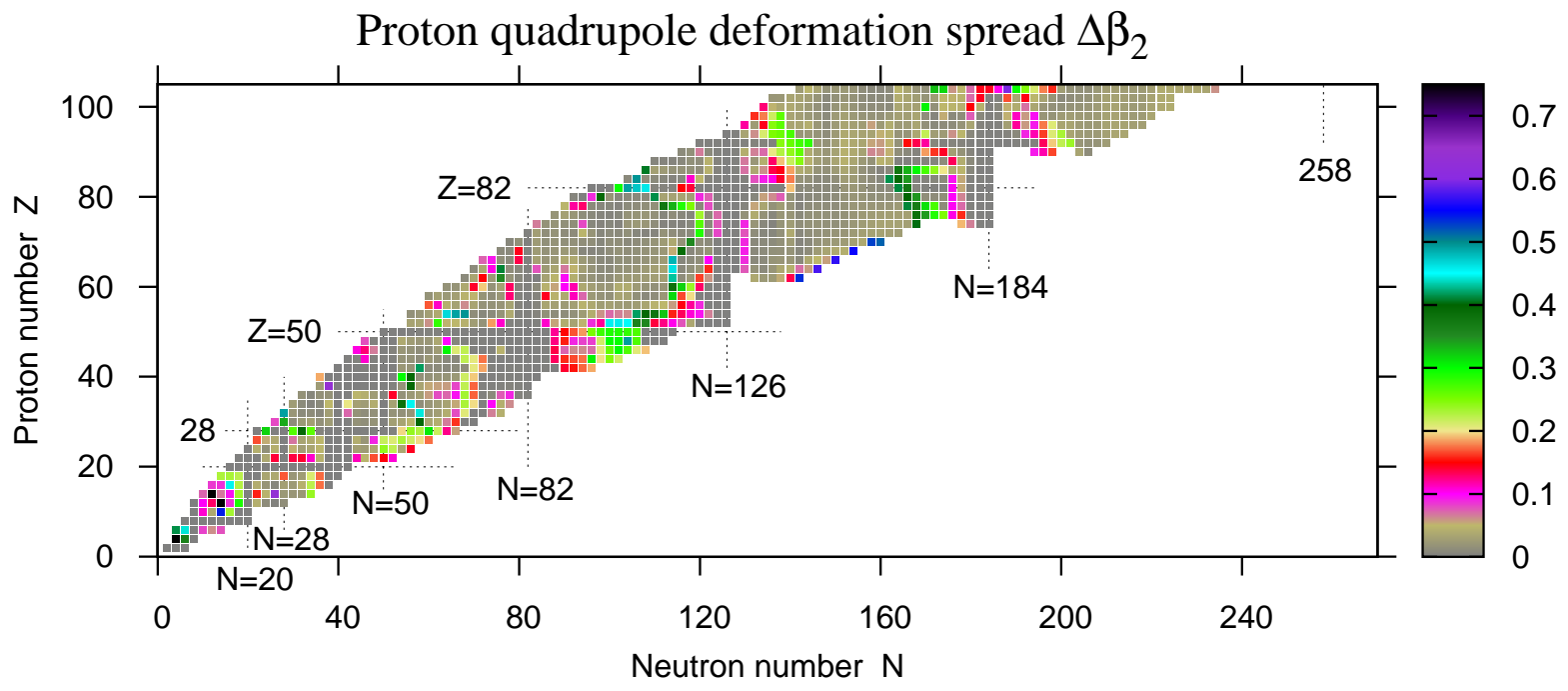

Figure 4. Proton quadrupole deformation spreads $\Delta \beta_{2}(Z, N)$ as a function of proton and neutron number. $\Delta \beta_{2}(Z, N)=\left|\beta_{2}^{\max }(Z, N)-\beta_{2}^{\min }(Z, N)\right|$, where $\beta_{2}^{\max }(Z, N)$ and $\beta_{2}^{\min }(Z, N)$ are the largest and smallest proton quadrupole deformations obtained with four employed CEDF's (NL3*, DD-ME2, DD-ME $\delta$ and DD-PC1) for the $(Z, N)$ nucleus at the ground state. From Ref. [31. See top panel of Fig. 7 for absolute values of the $\beta_{2}$-deformations calculated with DD-PC1.

Another process of interest which provides an important test of spectroscopic quality of the EDF is the rotation. The sequence of the states connected by stretched E2transitions are formed in the case of the rotation of deformed electric charge distribution. The evolution of the energies of these transitions and their strengths (in terms of $\mathrm{B}(E 2)$ ) with spin are frequently described in terms of the evolution of kinematic $J^{(1)}$ or/and dynamic $J^{(2)}$ moments of inertia and charge $\left(Q_{0}\right)$ or transition $\left(Q_{t}\right)$ quadrupole moments with rotational frequency $\Omega_{x}$. So far the systematic analysis of theoretical uncertainties in the description of these spectroscopic observables has only been performed in actinides and light superheavy nuclei in Refs. [20, 59] with the NL1 and NL3* CEDF's (see also discussion of ground state deformations in next paragraph). Some comparative results obtained with the NL1, NL3 and NLSH CEDF's are also available in the $A \sim 60\left({ }^{58} \mathrm{Cu}\right.$, ${ }^{60} \mathrm{Zn}$, and $\left.{ }^{62} \mathrm{Zn}\right)$ [60], $A \sim 150\left({ }^{143} \mathrm{Eu},{ }^{151} \mathrm{~Tb},{ }^{151} \mathrm{Dy}\right.$ and $\left.{ }^{152} \mathrm{Dy}\right)$ [37] and $A \sim 190\left({ }^{194} \mathrm{~Pb}\right.$ and ${ }^{194} \mathrm{Hg}$ [61] regions of superdeformation.

As illustrated in Fig. 4, theoretical uncertainties in the prediction of ground state proton quadrupole deformations are rather small for the regions of well deformed nuclei such as rare-earth and actinides. Moreover, experimental data on $\beta_{2}$ in these regions are well (typically within the experimental uncertainties) described by CDFT (see Sect. IX in Ref. [31]). Note that up to first backbending the rotation does not appreciably change the equilibrium deformation in these regions (see, for example, Fig. 7 in Ref. [44]) and that this change only weakly depends on CEDF. Thus, the results of Fig. 4 


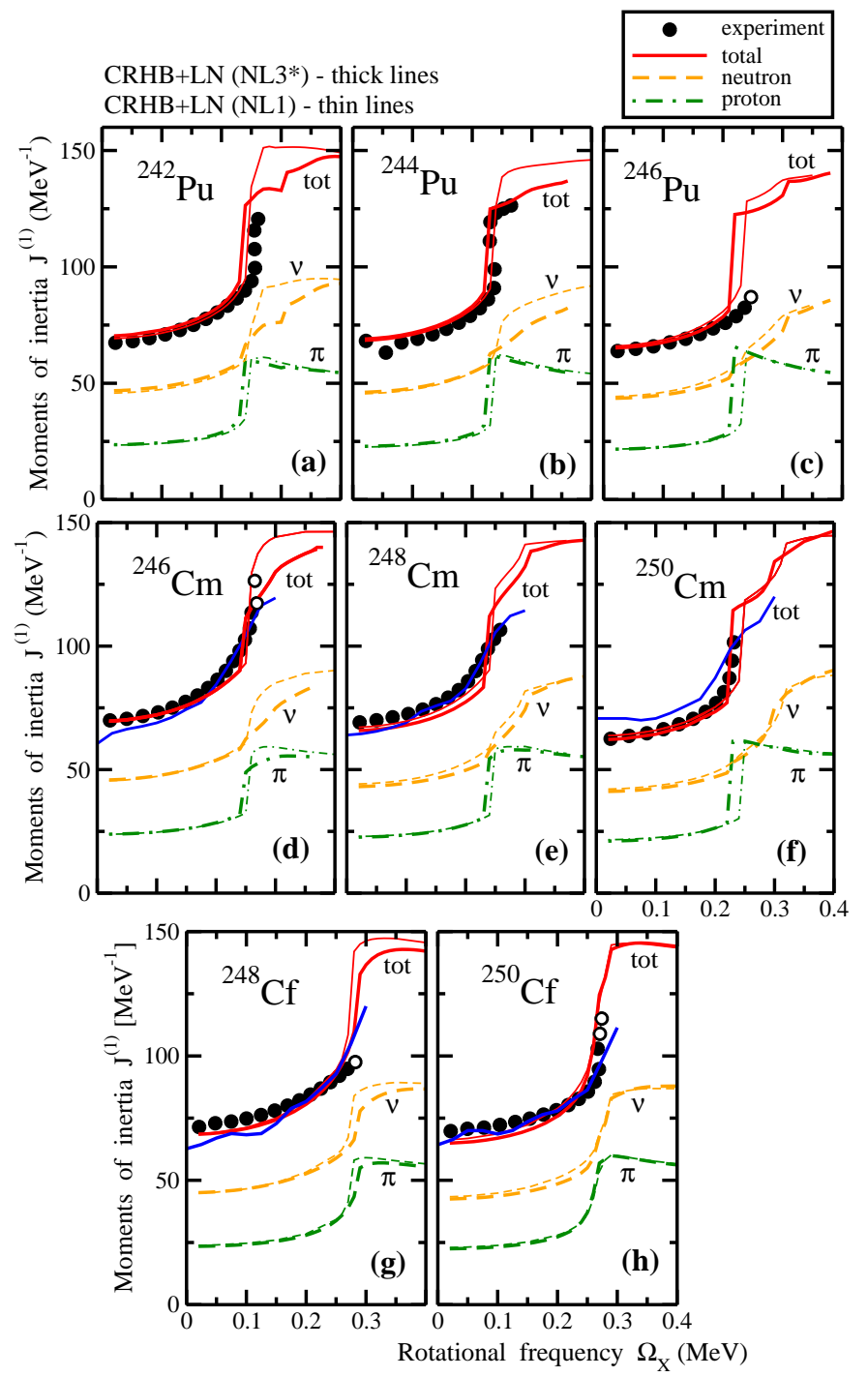

Figure 5. The experimental and calculated kinematic moments of inertia $J^{(1)}$ of ground state rotational bands in indicated nuclei as a function of rotational frequency $\Omega_{x}$. Proton and neutron contributions to the kinematic moment of inerta are presented. Open circles are used for tentative experimental points. See Ref. [59] for detailed comparison of these results.

strongly suggest that the deformations of ground state rotational bands up to first backbending in these regions are well described in CDFT irrespective of employed CEDF. This similarity of calculated deformations exists also at higher spin, where again the differences between the $Q_{t}$ values obtained with different CEDF's for the configuration of interest is typically within the experimental uncertainties (see also Refs. [37, 61] for results at superdeformation). Note that this result is strictly valid only for the configurations which have well pronounced minima in potential energy surfaces. The existing systematics of the calculated transition quadrupole moments in rotating nuclei [19, 60, 61, 62] shows that the CDFT well reproduces experimental data.

In addition to the deformation properties defining the strength of in-band E2- 
transitions, the spectroscopy of rotational bands shows up through the evolution of the moments of inertia with spin and band crossing features. Fig. 5 illustrates both that up to the band crossing region the gradual rise of the kinematic moment of inertia $J^{(1)}$ is well reproduced in the $\mathrm{CRHB}+\mathrm{LN}$ calculations and that the difference between two employed CEDF's (NL1 and NL3*) is rather marginal. Systematic comparison of such results (compare Figs. 9 and 10 in Ref. [20]) in actinides and light superheavy nuclei leads to the same conclusion.

The largest difference between the CEDF's shows up in the band crossing region. For example, the alignment of the $j_{15 / 2}$ neutrons in ${ }^{242,244} \mathrm{Pu}$ and ${ }^{246} \mathrm{Cm}$ proceeds in a gradual (sharp) way in the band crossing region in the CRHB+LN calculations with the NL3* (NL1) CEDF (Figs. 5 a, b and d). As a consequence, the alignment gain in the band crossing is also different in two CEDF's. However, for other nuclei shown in Fig. 5 these differences are smaller; sharp upbend takes place at sligthly different frequencies and the differences in the alignment gain are typically marginal. More examples of such differences between the results obtained with the NL1 and NL3* CEDF's can be found in the systematic study of Ref. [20]. The strength of the interaction between the $g$ and $S$ bands and the crossing frequency depends sensitively on the relative position of aligning high- $j$ orbital with respect to the quasiparticle vacuum [63]. Whether the alignment in the band crossing region proceeds in a gradual (gradual increase of $J^{(1)}$ ) or sharp (sharp upbend in $J^{(1)}$ ) way depends on whether the interaction strength between the $g$ and $S$ bands is strong or weak. Although the CRHB+LN calculations reproduce well the band crossings in ${ }^{242,244} \mathrm{Pu}$ and ${ }^{246,250} \mathrm{Cm}$, they fail to reproduce the gradual alignment in ${ }^{248} \mathrm{Cm}$. It follows from the comparison between theory and experiment that the interaction strength between the $g$ and $S$ bands shows variations with particle number which are not always reproduced in model calculations. The inaccuracies in the description of the single-particle states is one of possible reasons for this discrepancy between theory and experiment which decreases the predictive power of the models in the band crossing region.

Rotational properties of one-quasiparticle configurations in odd-mass nuclei provide an important information on the impact of odd particle/hole on alignment and pairing properties [20]. Considering that the energies of different deformed single-particle states with respect of the Fermi level and their relative energies depend on CEDF (see Sect. 3), it is important to estimate theoretical uncertainties in the description of rotational properties of odd-mass nuclei emerging from the use of different CEDF's. Such a systematic estimate has been performed in Ref. [20], and an illustrative example of ${ }^{237} \mathrm{U}$ is shown in Fig. 6,

For the $\nu 1 / 2[631]$ band in ${ }^{237} \mathrm{U}$, there is large separation between the $J^{(1)}$ values corresponding to the $(r= \pm i)$ branches at low frequency which gradually decreases and finally vanishes at high frequency (Fig. 6). This feature and the fact that the $(r=-i)$ branch has lower values of $J^{(1)}$ at low frequency are well reproduced in the calculations with the NL1 and NL3* CEDF's. However, the differences between the moments of inertia of these branches and the one in reference band of even-even nucleus 


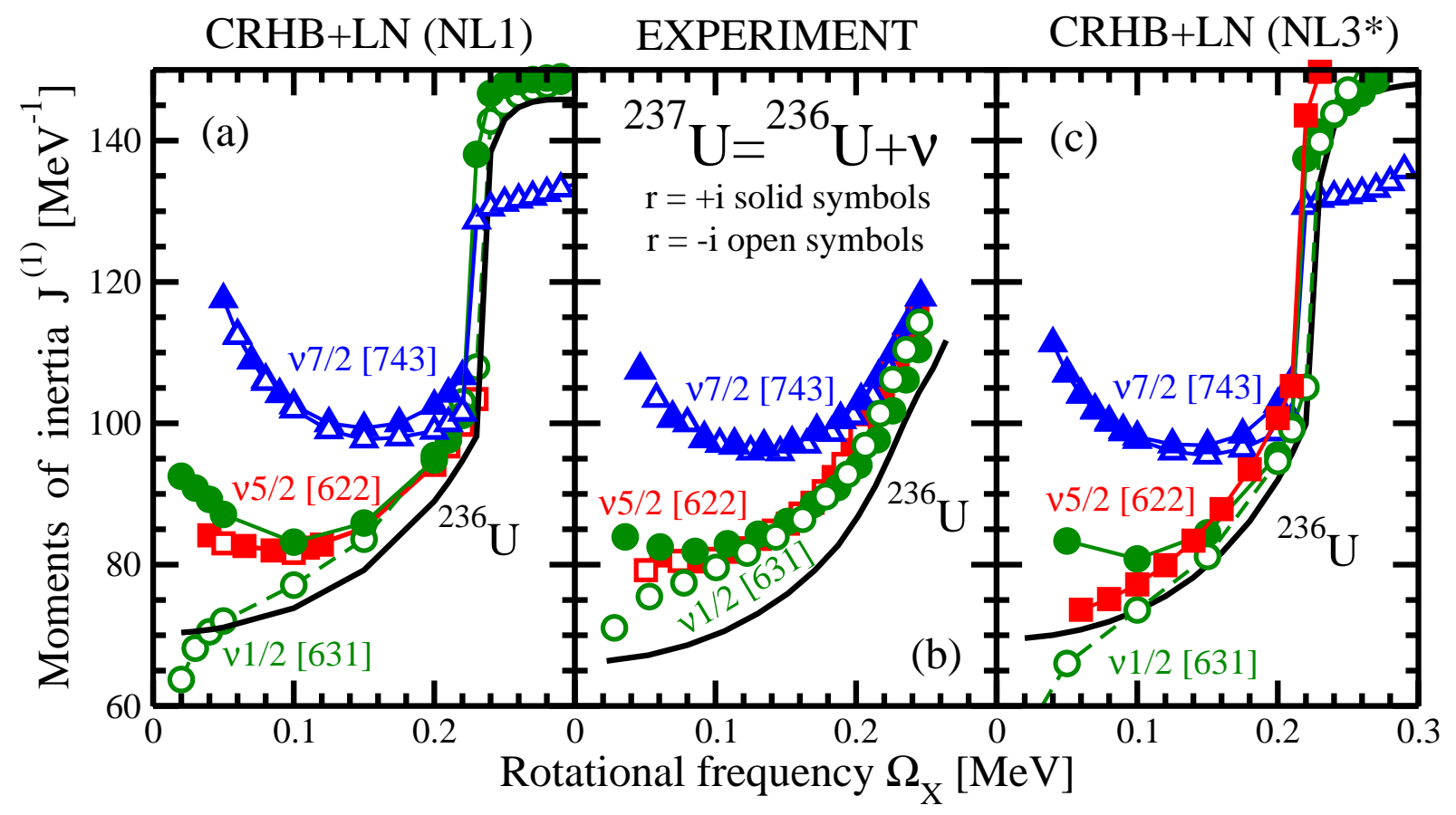

Figure 6. Calculated and experimental kinematic moments of inertia $J^{(1)}$ of the indicated one-quasiproton configurations in the ${ }^{237} \mathrm{U}$ nucleus and ground state rotational band in reference even-even ${ }^{236} \mathrm{U}$ nucleus. Experimental data are shown in the middle panel, while the results of the CRHB $+\mathrm{LN}$ calculations with the NL1 and NL3* CEDF's in the left and right panels, respectively. The same symbols/lines are used for the same theoretical and experimental configurations. The symbols are used only for the configurations in odd-mass nucleus; the ground state rotational band in reference even-even nucleus is shown by solid black line. From Ref. [20].

${ }^{236} \mathrm{U}$ are underestimated. The properties of two signature branches of the $\nu 7 / 2[743]$ rotational band such as the signature separation and its evolution with frequency, their absolute $J^{(1)}$ values and evolution with frequency as well as their relative properties with respect of reference band in ${ }^{236} \mathrm{U}$ are well reproduced in the calculations with both CEDF's. The $\nu 5 / 2[622]$ rotational band is signature degenerate. This feature is well reproduced in the $\mathrm{CRHB}+\mathrm{LN}(\mathrm{NL} 1)$ calculations. Only the $r=+i$ branch of this band has been obtained in the CRHB+LN(NL3*) calculations (Fig. 6r). However, the $\pi 5 / 2[523](r= \pm i)$ orbitals are signature degenerate in the frequency range of interest in the quasiparticle routhian diagram obtained with the NL3* CEDF. The absolute values of $J^{(1)}$ and their evolution with frequency are reproduced in model calculations. The NL1 CEDF somewhat better reproduces the properties of this band with respect of reference band in ${ }^{236} \mathrm{U}$ than the NL3* CEDF which underestimates the increase of the $J^{(1)}$ values due to blocking of the $\nu 5 / 2[622](r= \pm i)$ orbitals.

It is interesting to compare the $\mathrm{CRHB}+\mathrm{LN}$ calculations [20] with the results of the cranked shell model in which the pairing correlations are treated by a particlenumber conserving method (further $\mathrm{CSM}+\mathrm{PNC}$ ) 64]. In the CSM+PNC model, the parameters of the Nilsson potential were carefully adjusted to the experimental energies 
of deformed one-quasiparticle states of actinides and the experimental deformations were used. Despite that the average accuracy of the description of rotational properties in the band crossing region and below is similar in both models (Fig. 55) [59]. However, an accurate description of the rotational properties in CRHB $+\mathrm{LN}$ [20] is achieved in a more consistent way than in the CSM-NPC model. For example, contrary to CRHB+LN the accurate description of odd-mass nuclei in CSM+PNC model requires a different pairing strength as compared with even-even ones [64].

Despite the fact that single-particle energies are described less accurately in CDFT as compared with the MM method, other aspects of the single-particle motion such as (i) deformation polarization effects induced by particle or hole (measured in terms of relative [or differential] transition quadrupole moments $\Delta Q_{t}$ of two bands [37]) and (ii) alignment properties of single-particle orbital in rotating potential (measured by effective (relative) alignments [65] of two compared bands) are better described in CDFT. Indeed, the $\Delta Q_{t}$ values are well described in superdeformed rotational bands of the $A \sim 140-150$ mass region in CDFT [37, 66]; the average deviation from experiment is around $20 \%$. Similar (but somewhat less accurate because of the role of pairing) results have been obtained also in the $A \sim 130$ mass region of high- and superdeformation [67, 68]. The MM method based on the Nilsson potential describes deformation polarization effects also reasonably well. However, it suffers from the fact that these effects are not uniquely defined [69, 70]. Effective alignments are also on average better reproduced in the CDFT than in the cranked Nilsson-Strutinsky version of the MM approach based on phenomenological Nilsson potential, see comparisons presented in Refs. [17, 60, 71].

\section{Neutron drip lines}

The analysis of theoretical uncertainties in the prediction of the position of the neutron and proton drip-lines has recently attracted great interest [36, 75, 31] because of the possibility to estimate the number of nuclei which may exist in nature. Fig. 7 (bottom panel) shows the nuclear landscape which emerges from such an analysis performed in the framework of state-of-the-art non-relativistic and relativistic DFT's.

One can see that the largest uncertainties exist in the position of two-neutron drip line. Inevitably, the question about possible sources of these uncertainties emerges. For example, they were related to existing uncertainties in the definition of isovector properties of the EDF's (Ref. [36]). Indeed, the isovector properties of an EDF impact the depth of the nucleonic potential with respect to the continuum, and, thus, may affect the location of two-neutron drip line. However, inaccurate reproduction of the depth of the nucleonic potential exist in modern CEDF's also in known nuclei (see discussion in Sect. IVC of Ref. [11]). Thus, they alone cannot explain observed features. The observed differences in the prediction of the position of two-neutron drip line cannot also be explained by underlying nuclear matter properties of EDF's [31.

It was suggested in Ref. [75] that the position of two-neutron drip line sensitively depends also on the underlying shell structure and the accuracy of the description of the 

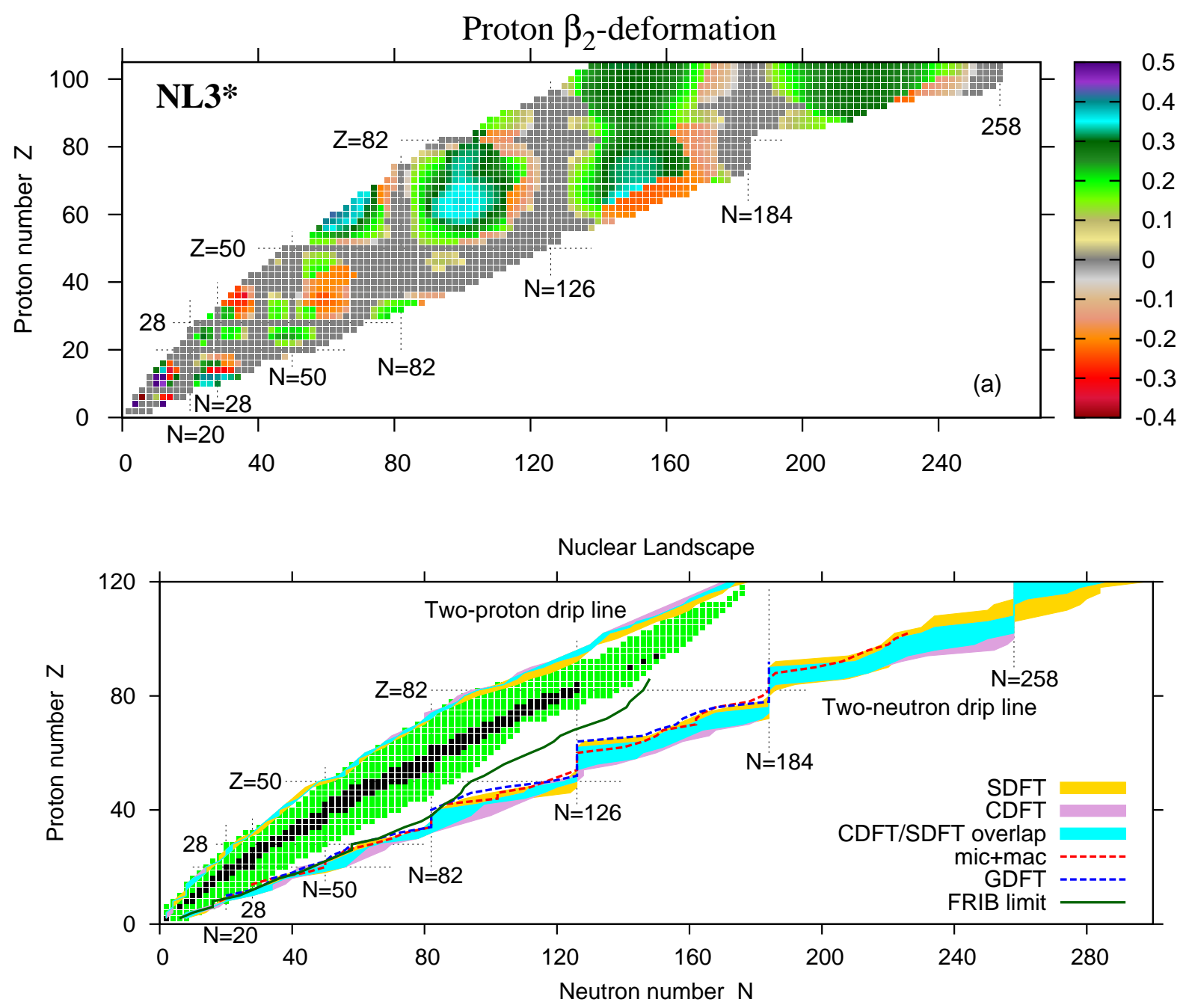

Figure 7. (top panel) Charge quadrupole deformations $\beta_{2}$ obtained in the RHB calculations with the NL3* CEDF. From Ref. 31. (bottom panel) Nuclear landscape as provided by state-of-the model calculations. The uncertainties in the definition of two-proton and two-neutron drip lines obtained in CDFT and Skyrme DFT (SDFT) are shown by shaded areas. They are defined by the extremes of the predictions of the corresponding drip lines obtained with different functionals. The blue shaded area shows the area where the CDFT and SDFT results overlap. Non-overlapping regions are shown by dark yellow and plum colors for SDFT and CDFT, respectively. The two-neutron drip lines obtained by microscopic+macroscopic (FRDM [72]) and Gogny D1S DFT 73 calculations are shown by dashed red and blue lines, respectively. Experimentally known stable and radioactive (including proton emitters) nuclei are shown by black and green squares, respectively. Green solid line shows the limits of nuclear chart (defined as fission yield greater than $10^{-6}$ ) which may be achieved with dedicated existence measurements at FRIB [74. Based on Fig. 4 of Ref. 75].

energies of the single-particle states. Indeed, the shell structure effects are clearly visible in the fact that for some combinations of $Z$ and $N$ there is basically no (or very little) dependence of the predicted location of the two-neutron drip line on the EDF [75, 31] (see bottom panel of Fig. 7). Such a weak (or vanishing) dependence, seen in all model 
calculations, is especially pronounced at spherical neutron shell closures with $N=126$ and 184 around the proton numbers $Z=54$ and 80, respectively. In addition, a similar situation is seen in the CDFT calculations at $N=258$ and $Z \sim 110$. This fact is easy to understand because of the large neutron shell gap at the magic neutron numbers in all DFT's. This is illustrated in Fig. 8 where the magic $N=184$ shell gap has a significant size of around $4 \mathrm{MeV}$ for all CEDF's. Note that only first four CEDF's of Fig. 8 were used in the definition of theoretical uncertainties in the position of two-neutron drip line in Fig. 7.

Theoretical uncertainties in the description of the energies of individual spherical orbitals shown in Fig. 8 are summarized in Fig. 9. They are substantial and in the majority of the cases they exceed $1 \mathrm{MeV}$; these uncertainties are below $1 \mathrm{MeV}$ only for three low- $j$ orbitals, namely, $4 p_{1 / 2}, 4 p_{3 / 2}$ and $3 f_{5 / 2}$. However, they are comparable with the ones observed in known nuclei (see the discussion in Sect. 2). These uncertainties definitely affect the position of two-neutron drip line and several factors discussed below play a role.

First, the comparison of bottom and top panels of Fig. 7 shows that there is a close correlation between the nuclear deformation at the neutron-drip line and the uncertainties in the prediction of this line. The regions of large uncertainties corresponds to transitional and deformed nuclei. Again this is caused by the underlying level densities of the single-particle states. The spherical nuclei under discussion are characterized by large shell gaps and a clustering of highly degenerate single-particle states around them. Deformation removes this high degeneracy of the single-particle states and leads to a more equal distribution of the single-particle states with energy.

Second, the large density of the neutron single-particle states close to the neutron continuum leads to a small slope of two-neutron separation energies $S_{2 n}$ as a function of neutron number in the vicinity of two-neutron drip line for medium and heavy mass nuclei (see Fig. 12 in Ref. [31]). As discussed in details in Sec. VIII of Ref. [31] this translates into (i) much larger uncertainties in the definition of the position of twoneutron drip line as compared with two-proton drip line and (ii) stronger dependence (as compared with two-proton drip line) of the predictions for the position of the twoneutron drip line on the accuracy of the description of the energies of the single-particle states.

Third, the position of the cluster of the states above the zero energy, the ordering of the single-particle states in this cluster and, in particular, the relative positions of low- $j$ and high- $j$ spherical orbitals (see Fig. 8) play an important role. This can be illustrated by the analysis of the $\operatorname{Rn}(Z=86)$ isotope chains, two-neutron drip lines of which are located at $N=206$ in the NL3* CEDF and at $N=184$ in the DD-ME2, DD-ME $\delta$ and DD-PC1 CEDF's (Table IV in Ref. [31]). The spectra shown in Fig. 8 will be used in this analysis; the increase of proton number from $Z=82(\mathrm{~Pb})$ up to $Z=86$ (Rn) leads to a more or less constant shift down (by approximately $400 \mathrm{keV}$ ) of the single-particle spectra. The strong dependence of the position of predicted neutron-drip line on the energies of the single-particle states is seen in the fact that the energies of 
the lowest positive energy states obtained with the NL3* and DD-ME2 CEDF's (two left columns in Fig. 8), which are expected to be active in the $N \geq 184$ nuclei, differ by only $160 \mathrm{keV}$. However, this difference alone cannot explain the twenty two (22 !!!) neutron difference in the predicted position of two-neutron drip line. It is clear that the presence of low-lying high-degeneracy $2 h_{11 / 2}$ orbital (which is also deformation-driving) at low energy in the NL3* CEDF is important for an extension of the nuclear landscape up to $N=206$. On the contrary, this orbital is located at higher energy in the DDCEDF's (Fig. 8). This together with higher energies of other positive energy states leads to the termination of nuclear landscape at $N=184$ in these CEDF's.

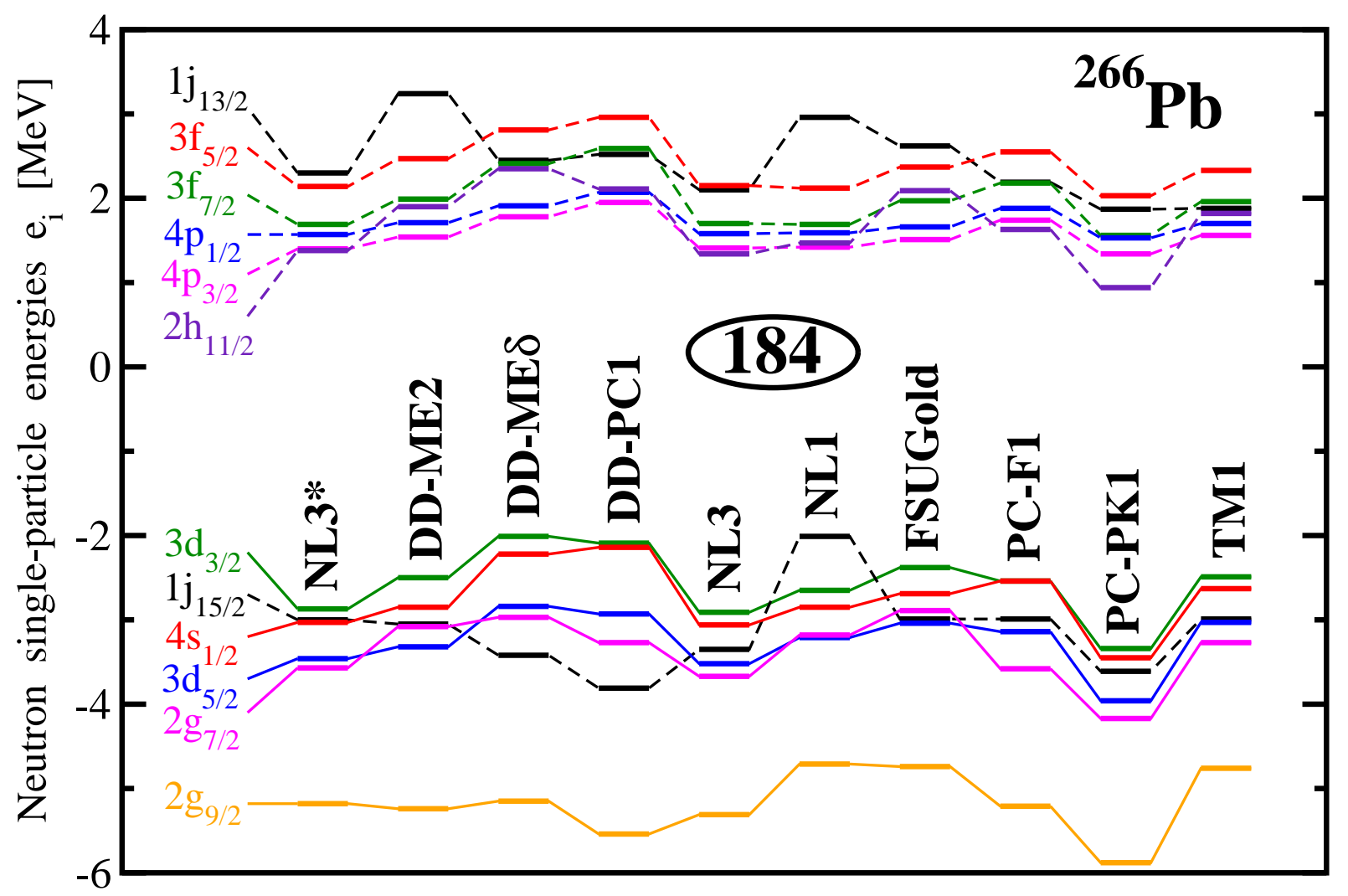

Figure 8. Neutron single-particle states at spherical shape in a ${ }^{266} \mathrm{~Pb}$ nucleus obtained with indicated CEDF's. Solid and dashed connecting lines are used for positive and negative parity states. Spherical gap at $N=184$ is indicated; all the states below this gap are occupied. For simplicity, only six lowest states above and six highest states below the gap are shown.

\section{Conclusion}

In the current manuscript, the question of spectroscopic quality of the description of physical phenomena in the framework of covariant density functional theory has been considered by analysing the accuracy and theoretical uncertainties in the description 


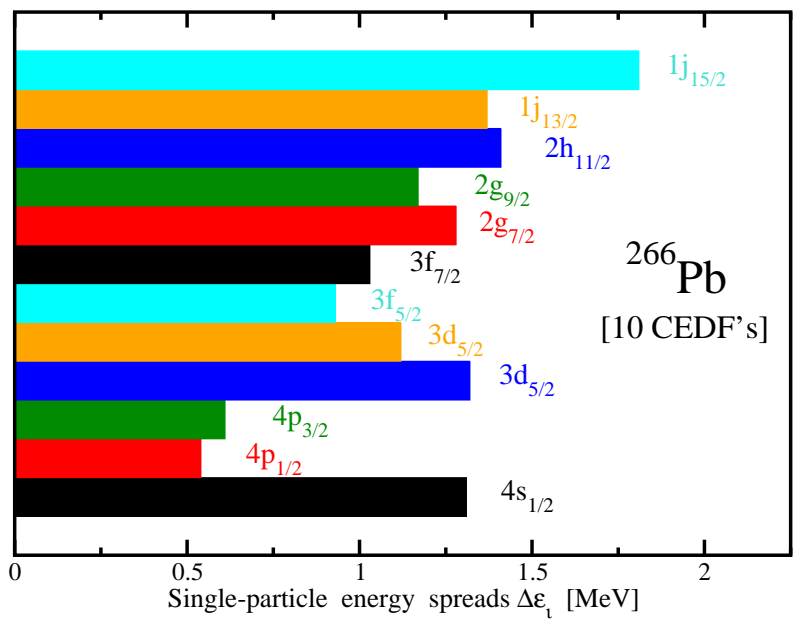

Figure 9. The spreads $\Delta \epsilon_{i}$ for indicated neutron single-particle states in ${ }^{266} \mathrm{~Pb}$. $\Delta \epsilon_{i}=\left|\epsilon_{i}^{\max }-\epsilon_{i}^{\min }\right|$, where $\epsilon_{i}^{\max }$ and $\epsilon_{i}^{\min }$ are the largest and smallest energies of a given single-particle state obtained with ten employed CEDF's for a given singleparticle state. The orbital angular momentum of the single-particle state increases on going from bottom to the top of the figure. Based on the results presented in Fig. 8 .

of different physical observables; these uncertainties are related to systematic errors of Ref. [30]. The following conclusions have been obtained:

- The current generation of the covariant energy density functionals has been obtained in the fitting protocols based on bulk and nuclear matter observables; no single-particle observables have been used in these protocols. Observed spreads in the predictions of the energies of the single-particle states in spherical and deformed nuclei when different CEDF's are used clearly indicate that such fitting protocols do not sufficiently constraint such observables. In medium and heavy-mass spherical nuclei relativistic particle-vibration coupling improves the description of the spectra. However, the remaining differences between theory and experiment clearly indicate missing physics and missing terms of CEDF's.

- To improve the description of the single-particle states new types of the fitting protocols including experimental data on the single-particle states are needed. At the current level of the development of CDFT, two types of the protocols could become feasible in near future. First, the protocols focused on spherical nuclei but which in addition to standard observables include the calculations of the spectra of few nuclei within the relativistic particle-vibration coupling model. Second, the protocols focused on deformed nuclei but which also require that for a large enough set of normal deformed odd nuclei the structure of the ground state is reproduced as a function of proton and neutron numbers. Such protocols avoid the effect of low effective mass of nucleon at the Fermi level since only the lowest in energy onequasiparticle state in each nucleus are used. Such states are only weakly affected by quasiparticle-phonon coupling [48], and, thus, can be reasonably well treated 
at the DFT level. At present, the ground states of odd-mass nuclei are correctly reproduced in the DFT calculations less frequently as compared with the MM ones [33, 76] and the improvement in that direction is highly desirable.

- Considering existing theoretical uncertainties in the description of the energies of the single-particle states it is important to understand which physical observables and in which situations are well or poorly described or predicted. Because of these uncertainties the predictive and descriptive power of the models decreases substantially (they can even give "false positive" or "false negative" signal on the existence of specific phenomenon) in the situations when the details of the potential energy surfaces (PES) sensitively depend on the underlying single-particle structure. As seen in Fig. 4 and discussed in details in Ref. [31] this takes place at zero spin in transitional nuclei, the PES of which is soft, and in nuclei characterized by prolateoblate shape coexistence. At higher spin, this takes place, for example, in chiral rotational bands, the PES of which are characterized by extremely shallow minima $(\sim 50 \mathrm{keV})$ [33].

- The uncertainties in the energies of the single-particle states become less important in nuclear systems with pronounced minima in the potential energy surfaces. These are, for example, well deformed nuclei in the rare-earth region and actinides or superdeformed structures across the nuclear chart. The rotational structures in the actinides were used to illustrate this feature. The calculated physical observables of actinides such as the moments of inertia and deformations only weakly depend on the selection of CEDF. The sensitivity of the results to the selection of CEDF is more pronounced in the paired band crossing region. Some aspects of the single-particle motion such as deformation polarization effects induced by particle or hole and the alignment properties of single-particle orbitals are better and more consistently described in the CDFT as compared with the models based on phenomenological Nilsson potential despite the fact that this potential better describes the energies of the single-particle states.

- The predicted position of two-neutron drip line in the majority of the isotope chains sensitively depends on the description of energies of the single-particle states in neutron-rich nuclei. It was illustrated in spherical nuclei that the uncertainties of such description are comparable in known and neutron-rich (near two-neutron drip line) nuclei. This fact strongly suggests that the improvement of the description of the energies of the single-particle states in known nuclei will reduce the uncertainties in the prediction of neutron-drip lines.

\section{Acknowledgements}

This work has been supported by the U.S. Department of Energy under the grant DE-FG02-07ER41459 and partially by an allocation of advanced computing resources provided by the National Science Foundation. The computations were partially performed on Kraken at the National Institute for Computational Sciences 
(http://www.nics.tennessee.edu/). The help of S. Agbemava and D. Ray in preparation of Figs. 3 and 7 and useful discussions with P. Ring are greatly appreciated. I would also like to thank H. Schatz for providing FRIB rates.

\section{References}

[1] Extended Density Functionals in Nuclear Structure Physics, Lecture Notes in Physics, edited by G. A. Lalazissis, P. Ring, and D. Vretenar (Springer-Verlag, Heidelberg, 2004) Vol. 641

[2] Cohen T D, Furnstahl R J and Griegel K 1992 Phys. Rev. C 451881

[3] Kaiser N and Weise W 2008 Nucl. Phys. A 80460

[4] Serot B D and Walecka J D 1997 Int. J. Mod. Phys. E6

[5] Furnstahl R J and Serot B D 2000 Nucl. Phys. A 673298

[6] Finelli P, Kaiser N, Vretenar D and Weise W 2006 Nucl. Phys. A 7701

[7] Kaiser N and Weise W 2010 Nucl. Phys. A 836256

[8] Furnstahl R J and Serot B D 2000 Nucl. Phys. A 671447

[9] Serot B D and Walecka J D 1986 Adv. Nucl. Phys. 161

[10] Ring P 1996 Prog. Part. Nucl. Phys. 37

[11] Litvinova E V and Afanasjev A V 2011 Phys. Rev. C 84014305

[12] Bender M, Rutz K, Reinhard P G, Maruhn J A and Greiner W 1999 Phys. Rev. C 60034304

[13] Bender M, Heenen P H and Reinhard P G 2003 Rev. Mod. Phys. 75121

[14] Koepf W and Ring P 1989 Nucl. Phys. A 49361

[15] Afanasjev A V and Abusara H 2010 Phys. Rev. C 81014309

[16] Hofmann U and Ring P 1988 Phys. Lett. B 214307

[17] Afanasjev A V and Ring P 2000 Phys. Rev. C 62 031302(R)

[18] Afanasjev A V and Abusara H 2010 Phys. Rev. C 82034329

[19] Vretenar D, Afanasjev A V, Lalazissis G A and Ring P 2005 Phys. Rep. 409101

[20] Afanasjev A V and Abdurazakov O 2013 Phys. Rev. C 88014320

[21] Schunck N, Dobaczewski J, McDonnell J, Moré J, Nazarewicz W, Sarich J and Stoitsov M V 2010 Phys. Rev. C 81024316

[22] Dobaczewski J and Dudek J 1995 Phys. Rev. C 521827

[23] Brockmann R and Toki H 1992 Phys. Rev. Lett. 683408

[24] Hofmann F, Keil C M and Lenske H 2001 Phys. Rev. C 64034314

[25] Serra M, Otsuka T, Akaishi Y, Ring P and Hirose S 2005 Prog. Theor. Phys. 1131009

[26] Hirose S, Serra M, Ring P, Otsuka T and Akaishi Y 2007 Phys. Rev. C 75024301

[27] Stoitsov M, Kortelainen M, Bogner S K, Duguet T, Furnstahl R J, Gebremariam B and Schunck N 2010 Phys. Rev. C 82054307

[28] Roca-Maza X, Viñas X, Centelles M, Ring P and Schuck P 2011 Phys. Rev. C 84054309

[29] Reinhard P G and Nazarewicz W 2010 Phys. Rev. C 81 051303(R)

[30] Dobaczewski J, Nazarewicz W and Reinhard P G 2014 J. Phys. G 41074001

[31] Agbemava S, Afanasjev A V, Ray D and Ring P 2014 Phys. Rev. C 89054320

[32] The free dictinionary, see http://encyclopedia2.thefreedictionary.com/Nuclear+Spectroscopy, this definition of Nuclear spectroscopy is based on "The Great Soviet Encyclopedia", 3rd Edition (1970-1979).

[33] Afanasjev A V and Shawaqfeh S 2011 Phys. Lett. B 706177

[34] König J and Ring P Phys. Rev. Lett. 71(19)

[35] Kortelainen M, Erler J, Nazarewicz W, Birge N, Gao Y and Olsen E 2013 Phys. Rev. C 88 031305(R)

[36] Erler J, Birge N, Kortelainen M, Nazarewicz W, Olsen E, Perhac A M and Stoitsov M 2012 Nature 486509

[37] Afanasjev A V, Lalazissis G and Ring P 1998 Nucl. Phys. A 634 
[38] Nikšić T, Vretenar D and Ring P 2008 Phys. Rev. C 78034318

[39] Zhao P W, Li Z P, Yao J M and Meng J 2010 Phys. Rev. C 82054319

[40] Litvinova E and Ring P 2006 Phys. Rev. C $\mathbf{7 3} 044328$

[41] Lalazissis G A, Karatzikos S, Fossion R, Arteaga D P, Afanasjev A V and Ring P 2009 Phys. Lett. B671 36

[42] D Tarpanov J Dobaczewski J T and Carlsson B G 2014 nucl-th arkhiv: 1405.4823v1

[43] Ring P and Schuck P 1980 The Nuclear Many-Body Problem (Springer-Verlag, Berlin)

[44] Afanasjev A V, Khoo T L, Frauendorf S, Lalazissis G A and Ahmad I 2003 Phys. Rev. C 67 024309

[45] Li J J, Long W H, Margueron J and Giai N V 2014 Physics Letters B 732169

[46] Litvinova E 2012 Phys. Rev. C 85 021303(R)

[47] Rodriguez-Guzman R, Sarriguren P and Robledo L M 2010 Phys. Rev. C 82 061302(R)

[48] Gareev F A, Ivanova S P, Soloviev V G and Fedotov S I 1973 Phys. Elem. Part. and At. Nucl. 4 357

[49] Soloviev V G Theory of complex nuclei, (Pergamon, 1976)

[50] Donati P, Døssing T, Shimizu Y R, Mizutori S, Bortignon P F and Broglia R A 2000 Phys. Rev. Lett. 84(19) 4317

[51] Jaminon M and Mahaux C 1989 Phys. Rev. C 40354

[52] Jain A K, Sheline R K, Sood P C and Jain K 1990 Rev. Mod. Phys. 62(2) 393

[53] Parkhomenko A and Sobieczewski A 2004 Acta Phys. Pol. 352447

[54] Abusara H, Afanasjev A V and Ring P 2010 Phys. Rev. C 82044303

[55] Lu B N, Zhao E G and Zhou S G 2012 Phys. Rev. C 85011301

[56] Prassa V, Nikšić T, Lalazissis G A and Vretenar D 2012 Phys. Rev. C 86024317

[57] Afanasjev A V, Abusara H and Ring P 2012 Int. J. Mod. Phys. E 211250025

[58] Afanasjev A V, Abusara H and Ring P 2013 Eur. Phys. J. Web of Conferences 6203003

[59] Afanasjev A V 2014 Phys. Scr. 89054001

[60] Afanasjev A V, Ragnarsson I and Ring P Phys. Rev. C 593166

[61] Afanasjev A V, Ring P and König J 2000 Nucl. Phys. A676 196

[62] Afanasjev A V 2013 chapter in the book "50 Years of Nuclear BCS", (World Scientific Publishing Co, Singapore, 2013), see also nuclear theory arkhive arXiv:1205.2134.

[63] Frauendorf S 2012 private communication

[64] Zhang Z H, He X T, Zeng J Y, Zhao E G and Zhou S G 2012 Phys. Rev. C 85014324

[65] Ragnarsson I 1993 Nucl. Phys. A 557 c167

[66] Nisius D et al. 1997 Phys. Lett. B 39218

[67] Laird R W et al. 2002 Phys. Rev. Lett. 88152501

[68] Matev M, Afanasjev A V, Dobaczewski J, Lalazissis G A and Nazarewicz W 2007 Phys. Rev. C 76034304

[69] Karlsson L B and Ragnarsson I 1998 Nucl. Phys. A 639654

[70] Aouad N E et al. 2000 Nucl. Phys. A 676155

[71] Afanasjev A V and Frauendorf S 2005 Phys. Rev. C 71064318

[72] Möller P, Nix J R, Myers W D and Swiatecki W J 1995 At. Data Nucl. Data Table 59185

[73] Delaroche J P, Girod M, Libert J, Goutte H, Hilaire S, Peru S, Pillet N and Bertsch G F 2010 Phys. Rev. C $\mathbf{8 1} 014303$

[74] Schatz H private communication (2014), see also https://groups.nscl.msu.edu/frib/rates/fribrates.html

[75] Afanasjev A V, Agbemava S, Ray D and Ring P 2013 Phys. Lett. B 726680

[76] Bonneau L, Quentin P, and Möller P 2007 Phys. Rev. C 76024320 